\title{
Abstracts
}

\section{The Gold Standard and the Great Depression}

This paper considers why political leaders and central bankers continued to adhere to the gold standard as the Great Depression intensified. We do not focus on the effects of the gold standard on the Depression, which have been documented elsewhere, but on the reasons why policy makers chose the policies they did. We argue that the mentality of the gold standard was pervasive and compelling to the leaders of the interwar economy. It was expressed and reinforced by the discourse among these leaders. It was opposed and finally defeated by mass politics, but only after the interaction of national policies had drawn the world into the Great Depression.

\section{'Das Kind' Revisited: Lord D'Abernon and German Security Policy I922-I925}

This article is a reassessment of F. G. Stambrook's well-known claim that Lord D'Abernon was the architect of German Locarno diplomacy. It suggests that Stambrook did not fully understand D'Abernon's relationship with the LutherStresemann government, and in particular it seeks to place German Locarno diplomacy within the wider context of German foreign policy in the period. It throws new light not only on the D'Abernon-von Schubert relationship but on the dynamic forces at work within the German Foreign Ministry. The article also contains a brief assessment of German relations with the United States.

\section{The Sour Fruits of Victory: Sterling and Security in Anglo-German Relations during the 1950 s and 1960 s}

By analysing the Anglo-German talks about financial compensation for the cost of British troops in West Germany, this article seeks to clarify the linkages between Britain's security and European and monetary policies. The ideological commitment of British politicians to a reserve currency role for sterling had a deep impact on Britain's relations to Europe, particularly the Federal Republic. By consistently 
threatening to cut its military commitment on the Continent and by minimising it to a question of financial expediency, London failed to draw any political capital from its troops. This became very visible in the field of European integration. The conflict on currency issues and troop deployment was a crucial element in preventing closer relations between the Federal Republic and the United Kingdom in the I950s and I960s.

\section{The Construction of Europe and the Concept of the Nation-State}

The construction of Europe is often teleologically addressed as a result of an unstoppable trend towards federalism. Another angle on this history gives access to another logic: that of a European kind of nation-state which considers European integration not as an element in its decline, but as a tool to reorganise its power. This new youth for the old nation-state was linked as much to the historical context of the I950s-I970s as to the specific rules of policy-making and to the economic regulation focus of the European Community.

\section{Extraits}

\section{L'Etalon-or et la Grande Crise}

Cet article cherche à comprendre pourquoi les gouvernants politiques et les dirigeants des banques centrales ont continué à adhérer à l'étalon-or alors que la Grande Crise se creusait. On ne s'attardera pas ici sur les conséquences du maintien de l'étalon-or sur la Crise, qui ont été explorés ailleurs, mais sur les raisons des choix faits par les décideurs. Notre hypothèse est que, chez les dirigeants économiques de l'entre-deux guerres, l'étalon-or était une valeur dominante et contraignante. Ceci était exprimé et renforcé par le discours en vigueur au sein de leur groupe. Cette idéologie de l'étalon-or fut finalement contestée et écartée au travers du processus démocratique, mais il fallut pour cela attendre que la conjugaison des politiques monétaires nationales ait entraîné le monde dans la Grande Crise.

\section{Un retour sur 'Das Kind': Lord d'Abernon et la politique de sécurité allemande}

Cet article est un réexamen de la thèse de F. G. Stambrook qui a fait de Lord d'Abernon l'architecte des accords de Locarno. L'article suggère que Stambrook n'a pas totalement compris la relation de d'Abernon avec le gouvernement LutherStresemann. Plus spécifiquement, on cherche ici à replacer la position allemande dans le plus large contexte de la politique étrangère allemande, notamment en ce qui concerne les relations avec les Etats-Unis. Ceci jette une nouvelle lumière non seulement sur la relation D'Abernon-Von Schubert, mais aussi sur les forces au travail au sein même du Ministère allemand des Affaires Etrangères. 


\section{Les fruits amers de la victoire: la livre sterling et la sécurité dans les relations anglo-allemandes dans les années I950 et 1960}

En analysant les discussions anglo-allemandes sur le remboursement des frais d'occupation, cet article s'attarde sur les liens entre les politiques monétaire, sécuritaire et européenne de la Grande-Bretagne. Le choix idéologique des politiciens britanniques, en faveur d'une livre-sterling forte, au statut de monnaie de réserve, fut d'une grande conséquence sur les relations de la Grande-Bretagne avec le reste de l'Europe, et en particulier avec la République Fédérale Allemande. Parce qu'elles menacèrent en permanence de réduire leur présence militaire, et parce qu'elles réduisirent la question à son aspect financier, les autorités britanniques ne purent tirer aucun bénéfice politique de la présence de laurs troupes. Ceci devint particulièrement visible autour de la question de la construction européenne. Le conflit sur les questions monétaires et sur l'entretien des troupes d'occupation fut un élément crucial qui empêcha des relations plus fortes entre la République Fédérale et le Royaume-Uni dans les années I950 et i960.

\section{La construction européenne et logiques de l'état-nation}

La construction européenne est souvent présentée de manière téléologique comme le résultat des seuls avancées et reculs d'un processus de fédéralisation inéluctable. Une lecture différente des faits marquants de cette histoire dévoile une autre logique: celle d'un Etat-nation européen voyant dans le processus d'intégration un instrument de redéploiement de sa puissance plus qu'un élément de déclin. Si le confinement de la Communauté dans le seul champ de la régulation économique et un mode de négociation décisionnel particulier ont joué un rôle certain, il ne faudrait pas négliger le poids de l'environnement historique des années 50-70 dans le phénomène de revitalisation et de relégitimation du vieil Etat-nation.

\section{Kurzfassungen}

\section{Der Goldstandard und die Große Depression}

Der Aufsatz erörtert, warum die politischen Führer und Bankiers am Goldstandard festhielten, während sich die Große Depression verschärfte. Im Mittelpunkt steht nicht die Wirkung des Goldstandards auf die Wirtschaftdepression, die bereits andernorts dokumentiert worden ist, sondern die Gründe, warum die politischen Entscheidungsträger sich so entschieden, wie sie das taten. Es wird die Meinung vertreten, daß die Mentalität des Goldstandards vorherrschte und den Wirtschaftspolitikern zwingend schien. Diese Einstellung fand im Diskurs Ausdruck und wurde dabei verstärkt. Schließlich wurde sie angegriffen und besiegt durch die Massenpolitik, allerdings erst, nachdem die nationalen Entscheidungen die Welt in die Große Depression gestürzt hatten. 


\section{'Das Kind' - eine erneute Betrachtung: Lord D'Abernon und die deutsche Sicherheitspolitik, I922-I925}

Der Aufsatz bewertet die bekannte Behauptung F. G. Stambrooks neu, daß Lord D'Abernon der Architekt der deutschen Locarnodiplomatie gewesen sei. Es wird darauf hingewiesen, daß Stambrook D’Abernons Beziehung zur Regierung LutherStresemann nicht ganz verstand. Insbesondere stellt der Aufsatz die deutsche Locarnopolitik in den weiteren Zusammenhang der deutschen Außenpolitik dieser Zeit. Hier wirft er sowohl neues Licht auf die Beziehung D'Abernons mit Schubert als auch auf die dynamischen Kräfte, die im deutschen Außenministerium wirkten. Der Aufsatz enthält außerdem eine knappe Einschätzung der deutsch-amerikanischen Beziehungen.

\section{Die saueren Früchte des Sieges: Sterling und Sicherheit in den deutsch- englischen Beziehungen der Fünfziger und Sechziger Jahre}

Der Aufsatz unternimmt es, die Verbindungen zwischen britischer Sicherheits-, Europe- und Geldpolitik zu klären, indem er die deutsch-englischen Verhandlungen über die Stationierungskosten analysiert. Die ideologische Festlegung britischer Politiker auf das Pfund als Reservewährung wirkte sich nachhaltig auf Großbritanniens Beziehungen zu Europa aus, insbesondere aber auf das Verhältnis zur Bundesrepublik Deutschland. Indem London immer wieder mit einer Reduzierung der militärischen Verpflichtungen auf dem Kontinent drohte und die Frage dabei auf finanzielle Zweckdienlichkeiten reduzierte, verschenkten die Regierungen die Chance, politisches Kapital aus ihren Truppen zu schlagen. Besonders deutlich wurde dies auf dem Feld der europäischen Integration. Der Konflikt um Währungsfragen und Truppenstationierung war ein entscheidender Faktor dafür, daß engere Beziehungen zwischen der Bundesrepublik Deutschland und dem Vereinigten Königreich in den Fünfziger und Sechziger Jahren nicht zustande kamen.

\section{Der Aufbau Europas und das Konzept des Nationalstaates}

Der Aufbau Europas wird häufig teleologisch dargestellt, als ob er das Ergebnis bloß von Fortschritten und (gelegentlichen) Rückschritten eines unausweichlichen Prozesses der Bundesstaatsbildung sei. Eine andere Lesart der Fakten, die diese Geschichte markieren, enthüllt aber eine ganz andere Logik: die des europäischen Nationalstaates, der im Integrationsprozeß ein Instrument sieht, um seine Macht wieder zu entfalten, darin aber kein Element des Niedergangs erblickt. Wenn sowohl die Beschränkung der Gemeinschaft allein auf das Feld der Wirtschaftregulierung als auch der besondere Verhandlungsmodus eine bestimmte Rolle spielten, darf dennoch das Gewicht nicht vergessen werden, das den historischen Umständen der Fünfziger bis Siebziger Jahre für das Phänomen eines wiederbelebten und neu legitimierten Nationalstaates zukam. 